\title{
1 Behavioral and biochemical effects of ethanol withdrawal in zebrafish
}

2 Suianny Nayara da Silva Chaves ${ }^{1}$, Gabriel Felício Rocha ${ }^{1}$, Bruna Patrícia Dutra Costa ${ }^{1}{ }^{4}$, Witallo

3 Etevaldo Araújo de Oliveira ${ }^{2}$, Monica Gomes Lima ${ }^{3,4}$, Diógenes Henrique de Siqueira Silva ${ }^{1,4}$, Caio

4 Maximino $^{1,4,5^{*}}$

5

6 Laboratório de Neurociências e Comportamento, Instituto de Estudos em Saúde e Biológicas,

7 Universidade Federal do Sul e Sudeste do Pará

$8 \quad{ }^{2}$ Escola Estadual de Ensino Médio Anísio Teixeira

$9 \quad{ }^{3}$ Laboratório de Neurofarmacologia e Biofísica, Campus VIII, Universidade do Estado do Pará

$10{ }^{4}$ Programa de Pós-Graduação em Biodiversidade e Biotecnologia - Rede BIONORTE

$11{ }^{5}$ Programa de Pós-Graduação em Neurociências e Comportamento, Universidade Federal do Pará

12

13

14

$15 *$ Corresponding author:

16 Caio Maximino

17 Laboratório de Neurociências e Comportamento - Instituto de Estudos em Saúde e Biológicas,

18 Universidade Federal do Sul e Sudeste do Pará, Unidade III

19 Av. dos Ipês, S/N, s/ CEP, Bairro Cidade Jardim, Marabá/PA, Brazil

20 cmaximino@unifesspa.edu.br 
21 Abstract: Chronic alcohol use induces adaptations and toxicity that can induce symptoms of

22 anxiety, autonomic hyperarousal, and epileptic seizures when alcohol is removed (withdrawal

23 syndrome). Zebrafish has recently gained wide attention as a behavioral model to study the

24 neurobehavioral effects of acute and chronic alcohol use, including withdrawal. The literature,

25 however, is very contradictory on findings regarding withdrawal effects, with some studies

26 reporting increased anxiety, while others report no effect. A meta-analytic approach was taken to

27 find the sources of this heterogeneity, and ethanol concentration during exposure and exposure

28 duration were found to be the main sources of variation. A conceptual replication was also made

29 using continuous exposure for 16 days in waterborne ethanol $(0.5 \%)$ and assessing anxiety-like

30 behavior in the light/dark test after $60 \mathrm{~min}$ withdrawal. Withdrawal was shown to reduce preference

31 for darkness, consistent with decreased anxiety, but to increase risk assessment, consistent with

32 increased anxiety. Animals were also subjected to the withdrawal protocol and injected with

33 pilocarpine in a sub-convulsive dose to assess susceptibility to epileptic seizure-like behavior. The

34 protocol was sufficient to increase susceptibility to epileptic seizure-like behavior in animals

35 exposed to ethanol. Finally, withdrawal also decreased catalase activity in the brain, but not in the

36 head kidney, suggesting mechanisms associated with the behavioral effects of ethanol withdrawal.

37 Keywords: Anxiety; Danio rerio; Ethanol withdrawal; Catalase activity; Epileptic seizures. 


\section{Introduction}

Chronic alcohol (ethanol, EtOH) use produces adaptations and toxicity that can lead to tolerance and dependence, manifested as physical and mental distress when EtOH is removed (withdrawal); symptoms of ethanol withdrawal include anxiety, insomnia, and autonomic hyperarousal (Krystal and Tabakoff, 2002). In more serious conditions, patients presenting this EtOH withdrawal syndrome can present perceptive changes, agitation, mental confusion, significant increases in autonomic arousal, and epileptic seizures (Gatch and Lal, 2001; Krystal and Tabakoff, 2002). The most serious condition involves delirium tremens and death by hyperthermia, cardiac arrhythmia, and complications from withdrawal-induced epileptic seizures (Longo and Schuckit, 2014). These symptoms also present with a typical time course, with marked signs of anxiety appearing as early as 6 hours after cessation of alcohol consumption, and epileptic seizures appearing from 12 to 48 hours after withdrawal (Trevisan et al., 1998). Since withdrawal symptoms are usually reduced after EtOH consumption, EtOH dependence can be maintained by negative reinforcement (Koob and Le Moal, 2008), and therefore investigating these motivational mechanisms could open new avenues for the treatment of EtOH consumption-related disorders. In animal models, EtOH withdrawal changes the excitability of neurons located in brain regions associated with defensive behavior, anxiety, and fear (Bonassoli et al., 2011; Chakravarty and Faingold, 1998; Long et al., 2007; Yang et al., 2003, 2002, 2001). Moreover, EtOH withdrawal also dysregulates the activity of the hypothalamus-pituitary-adrenal axis that modulates behavioral and endocrine responses to stress (Rasmussen et al., 2002). It makes sense, then, that the majority of animal models of EtOH withdrawal are focused on anxiety-like behavior, with consistent effects observed in rodent models such as the elevated plus-maze, light/dark box, social interaction test, and a drug discrimination assay using pentylenetetrazole (Gatch and Lal, 2001). Zebrafish (Danio rerio) is increasingly being considered as useful model organisms for studying both behavioral genetics and behavioral neuroscience, neuropsychopharmacology, and 
63 neurotoxicology (Bonan and Norton, 2015; Kalueff et al., 2012; Norton and Bally-Cuif, 2010;

64 Shams et al., 2018; Stewart et al., 2015). The main advantages associated with this species are its

65 low cost of acquisition and upkeep, ease handling, short lifespan, and readiness of reproduction in

66 laboratory environments (Gerlai, 2014; Kalueff et al., 2014). The relatively high degree of genetic,

67 neural, and endocrine homology with rodents and human beings is also cited as an advantage

68 (Kokel and Peterson, 2008). Zebrafish is also a good model for studying anxiety and stress, with

69 well-validated assays for novelty- and conflict-induced anxiety, social interaction, and antipredatory

70 behavior (Gerlai, 2010; Maximino et al., 2010; Oliveira, 2013). Importantly for EtOH withdrawal,

71 epileptic seizure-like behaviors were also characterized in the species (Hortopan et al., 2010),

72 although not yet in a context of EtOH withdrawal.

73 Zebrafish anxiety-like behavior has been used to demonstrate the effects of drug withdrawal,

74 including cocaine (López-Patiño et al., 2008a, 2008b), morphine (Cachat et al., 2010; Khor et al.,

75 2011; Wong et al., 2010), and EtOH (Tran et al., 2016). In the last case, the literature is

76 inconsistent, with some studies (e.g., Tran et al., 2015) reporting significant effects of withdrawal

77 on anxiety-like behavior, while others (e. g., Cachat et al., 2010) were unable to detect effects of

78 EtOH withdrawal. Procedural differences, such as assay type, strain, concentration during exposure,

79 or withdrawal duration, could be responsible for this difference.

One important consistency that is found in the literature regards results using the light/dark

81 test. For example, Benneh et al. (2016) and Mathur and Guo (2011) found no effect of withdrawal

82 on dark preference, while Holcombe et al. (2013) found that zebrafish subjected to EtOH

83 withdrawal reversed their preference, spending more time in the white compartment instead of the

84 black compartment. The light/dark test is conceptually different from the novel tank test (one of the

85 most commonly used assays in zebrafish withdrawal research) in that an approach-avoidance

86 conflict appears to underline behavior in the light/dark test, while escape from the top appears to

87 motivate behavior in the novel tank test (Maximino et al., 2012); a recent metanalysis (Kysil et al, 
88 2017) also suggested that the light/dark test is more sensitive to pharmacological treatments than the

89 novel tank test. The discrepancies in the literature regarding the effects of EtOH withdrawal on both

90 tests could represent different underlining neurobiological bases, or methodological differences.

91 In addition to these behavioral endpoints, neurochemical analyses and oxidative stress

92 assays were also reported in zebrafish models of EtOH withdrawal (Müller et al., 2017; Tran et al.,

93 2015b). After exposure to $0.5 \%$ EtOH for 22 days, increases in brain levels of dopamine, serotonin,

94 and aspartate were observed with $60 \mathrm{~min}$ withdrawal (Pan et al., 2012). After exposure for 8 days to

$951 \% \mathrm{EtOH}$ (for 20 min per day), decreases in superoxide dismutase and catalase activity and

96 consequent increases in oxidative stress were observed (Müller et al., 2017). While a mechanistic

97 explanation is still lacking, these results are congruent with the hyperexcitability and EtOH-induced

98 neurotoxicity observed in other models (Krystal and Tabakoff, 2002; Zenki et al., 2014).

99 The aim of the present work is to study the heterogeneity of effects of EtOH withdrawal on

100 zebrafish anxiety-like behavior in the literature, by applying meta-analytical techniques. Moreover,

101 the present work attempts a conceptual replication of findings on EtOH withdrawal by assessing the

102 effects of a withdrawal protocol on behavior in the light/dark test. It also attempts to expand the

103 range of endpoints used in withdrawal research by using a chemically-induced epileptic seizure-like

104 behavior model with sub-convulsive doses. Finally, the present work also attempted to replicate

105 previous research on the effects of $\mathrm{EtOH}$ withdrawal on the activity of the enzyme catalase in the

106 brain and head kidney. This manuscript is a complete report of all the studies performed to test the

107 effect of ethanol withdrawal on anxiety-like and convulsive-like behavior in zebrafish. We report

108 how we determined our sample size, all data exclusions (if any), all data transformations, and all

109 measures in the study (Simmons et al., 2012).

110

111 2. Methods

112 2.1 Systematic review and metanalysis 
113 The protocol for the meta-analysis was pre-registered in the CAMARADES-NC3Rs

114 Preclinical Systematic Review \& Meta-analysis Facility (SyRF) database

115 (https://drive.google.com/file/d/0B7Z0eAxKc8ApUjcyQjhwVnFjRFE/view?usp=sharing). No

116 modification from the pre-registered protocol was made. Article with the descriptors 'ethanol

117 withdrawal' and 'zebrafish' were searched for in PubMed (https://www.ncbi.nlm.nih.gov/pubmed),

118 using a search filter optimized to finding studies on animal experimentation on PubMed (Hooijmans

119 et al., 2010). Bibliographic data (including DOI, publication date, title, and abstract) from the

120 studies identified in the systematic review were exported to a spreadsheet. Each article from the list

121 was reviewed in four levels of detail (title, abstract, full text, and a detailed revision of the

122 experimental design) in order to determine its eligibility to meta-analysis. Following Mohammad et

123 al. (2016), studies should include (1) primary behavioral data obtained in tests for anxiety-like

124 behavior in zebrafish (light/dark test, novel tank test, antipredator responses, shoaling responses);

125 (2) reporting of appropriate controls; and (3) reporting of at least sample sizes and summary

126 statistics (central tendency and dispersion measures) for control and withdrawal groups. While the

127 light/dark and novel tank tests, as well as antipredator responses, straightforwardly represent

128 anxiety-like behavior, shoaling has been included because it is sensitive to manipulations which

129 increase anxiety and/or fear in zebrafish (Green et al., 2012), suggesting a defensive component.

130 When an experiment evaluated the effects of drugs or other interventions on withdrawal syndrome-

131 like effects, only control and $\mathrm{EtOH}$ withdrawal groups were considered, and data on intervention

132 effects was not analyzed; e.g., while Pittman and Hylton (2015) assessed the effects of fluoxetine

133 and ketamine on withdrawal-induced anxiogenesis, these effects were not assessed in the

134 metanalysis. Possible confounds in relation to the role of development were reduced by excluding

135 studies which were not performed on adult fish.

The following data were extracted from each included study: identification (DOI, authors,

137 publication year); strain/phenotype; concentration of ethanol during exposure; duration of EtOH 
138 treatment; duration of withdrawal; behavioral test that was used; means and standard deviations, as

139 well as a test statistics and degrees of freedom; and sample sizes $(N)$ for each group. Data, which

140 were represented graphically, were extracted from figures using PlotDigitizer

141 (http://plotdigitizer.sourceforge.net/). When multiple dependent variables were reported, only the

142 primary endpoint was used (time on white, time on bottom, inter-fish distance, and distance from

143 stimulus). While there is considerable variation in the effects of interventions on these tasks - and

144 indeed variables such as erratic swimming or freezing can be more sensitive to certain treatments -,

145 the literature usually refers to time on white and time on bottom as the primary endpoints. Distance

146 from stimulus was used as a primary endpoint for the shoaling and antipredator behavior

147 experiments made by Robert Gerlai's group, given that it indicates increased shoaling (decreased

148 distance to shoal stimulus) or predator avoidance (increased distance to predator stimulus). All

149 estimates were transformed to standardized mean differences (SMD), corrected for its positive bias

150 (Hedges and Olkin, 1985), with unbiased estimates of sampling variances and confidence intervals

151 at the $95 \%$ level, $I^{2}$ and $\tau^{2}$ heterogeneity values, and p-values using a mixed-effects model, with

152 concentration, exposure duration, and withdrawal duration used as moderators. Differently from the

153 other endpoints, decreased distances to the shoal stimulus or decreased inter-fish distances indicate

154 less anxiety, and the SMDs for these cases were transformed by multiplying by - 1 (Vesterinen et al.,

155 2014). Influential case diagnostics was made by inspecting plots for externally standardized

156 residues, DFFITS values, Cook's distances, covariance ratios, estimates of $\tau^{2}$ and test statistics for

157 residual heterogeneity when each study is removed in turn, hat values, and weights for each study

158 included in the analysis. Publication bias was assessed by inspection of a contour-enhanced funnel

159 plot, with contours at the $90 \%, 95 \%$ and $99 \%$ confidence intervals. Moreover, funnel plot

160 asymmetry was analyzed using a meta-regression test, with total samples size as predictor (Egger et

161 al., 1997). Observed power for each study was calculated based on effect sizes, sample sizes and

162 standard deviations, and fitted against SMDs by a generalized additive model with Gaussian curve 
163 family and identity link function. Finally, a sensitivity analysis was performed by adding study

164 quality, assessed using SYRCLE's Risk of Bias (RoB) tool (Hooijmans et al., 2014), in the meta-

165 regression model. The meta-analysis was made with the metafor package from $\mathrm{R}$ (Viechtbauer, 166 2010). susceptibility in zebrafish 
187 manipulations minimized their potential suffering of animals, and followed Brazilian legislation

188 (Conselho Nacional de Controle de Experimentação Animal - CONCEA, 2017). Animals were used

189 for only one experiment and in a single behavioral test, to reduce interference from apparatus

190 exposure.

The EtOH exposure and withdrawal regimen was adapted from Gerlai et al. (2009) $\square$. In

194 brief, animals were exposed to increasing EtOH concentrations (0.125\%-0.5\%), dispersed on the

195 tank water, for 16 days, therefore decreasing mortality that is associated with prolonged exposure to

196 EtOH in zebrafish. Concentrations doubled every four days, reaching a final concentration of $0.5 \%$

197 (v/v). Animals were kept in this final concentration for 4 days. Another group was exposed to the

198 same manipulation, without EtOH exposure; animals were randomly allocated to treatments via

199 generation of random numbers using the randomization tool in http://www.randomization.com/.

200 Caretakers were blinded for treatment. After treatment, animals were transferred to a tank with

201 system water for $60 \mathrm{~min}$ (withdrawal stage). All animals were included in the experiments, and no

202 gross physical abnormalities were observed during the exposure period. To control for effects of

203 chronic EtOH exposure that are not attributable to withdrawal, two additional groups of 8 animals

204 were exposed as above and transferred, without an withdrawal stage, to the light/dark tank.

After EtOH withdrawal, animals (10 animals in the control group, 14 in the withdrawal

208 group) were individually tested in a tank $(15 \mathrm{~cm}$ height $\mathrm{X} 10 \mathrm{~cm}$ width $\mathrm{X} 45 \mathrm{~cm}$ length) that was

209 divided in half into a black compartment and a white compartment. The tank was made of matte

210 acrylic. The apparatus was illuminated from above by two fluorescent $25 \mathrm{~W}$ lamps, which produced 
211 an average of 270 lumens above the tank (light levels were measured using a hand photometer). The

212 tank contained sliding doors that defined a central compartment in which the animal was positioned

213 for a 3-min acclimation. After this stage, the sliding doors were removed, allowing the animal to

214 freely explore the apparatus for $15 \mathrm{~min}$.

215 The order with which animals were exposed to the tank was randomized and balanced

216 across treatments. Experimenters were blinded to treatment. Videos were manually transcribed by

217 two observers, blinded to treatment, using X-Plo-Rat (https://github.com/lanec-unifesspa/x-plo-rat).

218 The following variables were analyzed: time on the white compartment (s); transitions to white;

219 total locomotion on white number of virtual $4.5 \mathrm{~cm}^{2}$ squares crossed by the animal in the

220 compartment); mean duration of entries in the white compartment (total duration divided by the

221 number of transitions); time freezing (s); number of erratic swimming events; time in thigmotaxis

222 (s); number of risk assessment events. Operational definitions of these endpoints can be found in

\section{Table 1.}

Data were analyzed via approximative two-sample Fisher-Pitman permutation tests with

22510.000 Monte-Carlo re-samplings. The data analyst was blinded to treatment by cell scrambling;

226 after analysis, data was unblinded. Data are presented using individual dot plots combined with

227 summaries of mean and bootstrapped confidence intervals at 95\% level. Standardized mean

228 differences with unbiased variance estimates were calculated using the R package 'metafor'. Post-

229 hoc (observed) power was calculated based on an approximation of the T-test, with two-tailed

230 hypotheses. All analyses and graphs were made using R version 3.3.0 and packages 'ggplot2' and 231 'coin'. 
235 pilocarpine. This dose has been shown to be insufficient to produce clonic and tonic-clonic epileptic

236 seizure-like behavior in adult animals (Pinto, 2015). Fifteen minutes after injection, animals were

237 individually transferred to $1.5 \mathrm{~L}$ tanks, and filmed for $15 \mathrm{~min}$ to analyze the profile of epileptic

238 seizure-like behavior. Epileptic seizure-like behaviors were scored according to Mussulini et al.

239 (2013), as in Table 2.

Score 4 is the minimum behavioral phenotype that can be considered epileptiform

241 (Mussulini et al., 2013); therefore, the latency to reach Score 4 was considered as the main endpoint

242 for epileptic seizure-like behavior. Latencies were analyzed by fitting a Kaplan-Meier model to 243 survival curves, using the R package 'survival'.

A separate group of 12 animals were used in this experiment. Animals were subjected to the exposure and withdrawal regimen described in 2.2.1. Sixty minutes after withdrawal, animals were

248 euthanized in cold water followed by spinal section, and their brains and head kidneys were 249 dissected. Catalase activity in those organs was measured using the rate of disappearance of $\mathrm{H}_{2} \mathrm{O}_{2}$ 250 spectrophotometrically, following the method described by Aebi (1984). Within-laboratory 251 validation yielded a linearity of $\mathrm{r}^{2}=0.9849$, and intermediary repeatability of 0.2364 (IC95\% $252[0.0042,0.4686]$; Horwitz ratio). Enzyme activity was corrected by protein levels, quantified by the 253 Bradford method. Differences between groups were analyzed using Approximative Two-Sample 254 Fisher-Pitman Permutation Tests.

\section{3. Results}

\section{3.1. Metanalysis}


To evaluate the effect of EtOH withdrawal on zebrafish anxiety-like behavior, we applied a

259 mixed-effects meta-regression model on the results from the systematic review. Characteristics

260 from the articles found in the systematic review can be found in Table 3; raw data and analysis

261 scripts for this metanalysis can be found in our GitHub repository (https://github.com/lanec-

262 unifesspa/etoh-withdrawal/tree/master/metanalysis). EtOH concentrations ranged from $0.25 \%$ to

$2633 \% \mathrm{v} / \mathrm{v}$ (median 1\%); exposure durations ranged from 7 to 63 days (median 14 days), and

264 withdrawal durations ranged from 1 to $1.512 \mathrm{~h}$ (median 48 h). In general, a high risk of bias was

265 observed, since most studies did not report blinding or random allocation (https://github.com/lanec-

266 unifesspa/etoh-withdrawal/blob/master/metanalysis/etoh-withdrawal-metanalysis-rob.csv).

267 Behavioral test, strain/phenotype, EtOH concentration, exposure duration, and withdrawal duration

268 were used as moderators. Results from this analysis are presented in the forest plot found in Figure

269 1A. Residual heterogeneity was estimated as $\tau^{2}=0.1667$, significantly high $\left(\mathrm{QE}_{[\mathrm{df}=11]}=22.6338, p\right.$

$270=0.0199)$, suggesting that although about $88 \%$ of the total heterogeneity can be explained by

271 including the five moderators in the model, other factors might influence the effect. After applying

272 a permutation test with 1.000 replications, a significant effect of exposure duration $(p=0.029)$ and

273 EtOH concentration $(p=0.001)$ were found, but the other mediators did not affect withdrawal-like

274 behavior (Figures 1B-1D). The contour-enhanced funnel plot (Figure 1E) suggest that most studies

275 failed to reach statistical significance, with only one study falling in the 95\% CI range, and one in

276 the 99\% CI range. Egger's test on this funnel plot did not suggest publication bias $\left(\mathrm{t}_{[\mathrm{df}}=10\right]=0.439$,

$277 p=0.67)$. An analysis of influential observations suggests that the Mathur and Guo (2011) study on

278 the effects on the NTT with the longer withdrawal duration and the Dewari et al. (2016) study

279 produced most residual heterogeneity (Figure S1). Absolute SMDs were significantly explained by

280 observed power (slope $=1.2819, p=0.0174$; Figure S2). Finally, sensitivity analysis suggested that

281 study quality did not influence results, as including RoB scores in the model did not improve fit 
282 (model without RoB: $\mathrm{AIC}=46.4816, \mathrm{BIC}=50.88=585$; model with $\mathrm{RoB}:$ AIC $=47.2908, \mathrm{BIC}=$ 283 50.9218).

Withdrawal increased time on white $\left(Z=2.1207, p=0.0261 ; \mathrm{SMD}_{\mathrm{UB}}=-0.995 ;\right.$ observed

power $=0.6414$; Figure 2A), without affecting entry duration $\left(\mathrm{Z}=0.92305, p=0.4301 ; \mathrm{SMD}_{\mathrm{UB}}=-\right.$

0.392; observed power $=0.147$; Figure 2B $)$. Withdrawal did not increase erratic swimming $(Z=$

1.9389, $p=0.0564, \mathrm{SMD}_{\mathrm{UB}}=0.890$; observed power $=0.5467$; Figure 2C), but it increased risk

assessment $\left(Z=1.9895, p=0.0405, \mathrm{SMD}_{\mathrm{UB}}=0.918\right.$; observed power $=0.5724$; Figure 2D $)$.

Freezing $\left(Z=0.8082, p=0.7003, \mathrm{SMD}_{\mathrm{UB}}=0.286\right.$; observed power $=0.098 ;$ Figure $\left.2 \mathbf{E}\right)$ and

thigmotaxis $\left(Z=0.41291, p=0.7024, \mathrm{SMD}_{\mathrm{UB}}=-0.2133\right.$; observed power $=0.0718$; Figure $\left.2 \mathrm{~F}\right)$

were unaffected. As for motor effects, transitions to white were not affected by withdrawal $(\mathrm{Z}=$ $0.91765, p=0.3763, \mathrm{SMD}_{\mathrm{UB}}=0.39 ;$ observed power $=0.1469 ;$ Figure 2G), but total locomotion was higher in animals exposed to withdrawal $\left(\mathrm{Z}=2.5965, p=0.0034, \mathrm{SMD}_{\mathrm{UB}}=1.31\right.$; observed power $=0.863$; Figure 2H).

To control for effects of chronic exposure, independent groups were chronically exposed to $\mathrm{EtOH}(0.5 \%)$, and tested in the light/dark test immediately after the last exposure (i.e., without withdrawal). Animals exposed to $\mathrm{EtOH}$ spent more time in the white compartment $(\mathrm{Z}=-$ 1.9535. $p$

$300=0.0467 ; \mathrm{SMD}_{\mathrm{UB}}=-1.033 ;$ observed power $=0.482 ;$ Figure $\left.\mathrm{S} 3 \mathrm{~A}\right)$, but did not show changes in 301 entry duration $\left(Z=-1.264, p=0.2632 ; \mathrm{SMD}_{\mathrm{UB}}=0.611\right.$; observed power $=0.204$; Figure $\left.\mathrm{S} 3 \mathrm{~B}\right)$.

302 Erratic swimming was decreased by chronic EtOH treatment $\left(\mathrm{Z}=2.521, p=0.01 ; \mathrm{SMD}_{\mathrm{UB}}=1.516\right.$;

303 observed power $=0.803$; Figure S3C), but no changes were observed on risk assessment $(\mathrm{Z}=0.478$, $304 p=0.793 ; \mathrm{SMD}_{\mathrm{UB}}=0.212 ;$ observed power $=0.058 ;$ Figure $\left.\mathrm{S} 3 \mathrm{D}\right)$ or freezing $(\mathrm{Z}=0.393, p=0.736$; $305 \mathrm{SMD}_{\mathrm{UB}}=0.180$; observed power $=0.052$; Figure $\left.\mathrm{S} 3 \mathrm{E}\right)$. Thigmotaxis was decreased by chronic 
306 EtOH $\left(\mathrm{Z}=2.2879, p=0.012 ; \mathrm{SMD}_{\mathrm{UB}}=1.295\right.$; observed power $=0.671$; Figure S3F $)$ Neither

307 transitions to white $\left(\mathrm{Z}=-1.790, p=0.074 ; \mathrm{SMD}_{\mathrm{UB}}=-0.922 ;\right.$ observed power $=0.402 ;$ Figure $\left.\mathrm{S} 3 \mathrm{G}\right)$

308 nor total locomotion $\left(\mathrm{Z}=-1.643, p=0.097\right.$; $\mathrm{SMD}_{\mathrm{UB}}=-0.829$; observed power $=0.336$; Figure

$309 \mathrm{~S} 3 \mathrm{H})$ were changed by chronic treatment with EtOH.

313 after $150 \mathrm{mg} / \mathrm{kg}$ pilocarpine, replicating findings from Pinto (2015); therefore, this dose is indeed

314 sub-convulsive in zebrafish. All animals from the withdrawal group exhibited Score IV epileptic

315 seizure-like behaviors after $150 \mathrm{mg} / \mathrm{kg}$ pilocarpine; after applying a log-rank model for the 316 differences in latencies to Score IV, a significant difference was seen in the withdrawal group $\left(\chi^{2}\right.$ [df $=$ 317 1] $=8.8, p=0.00308$; Figure 3). This data can be found in our GitHub repository 318 (https://github.com/lanec-unifesspa/etoh-withdrawal/tree/master/seizure).

\subsection{Effects of EtOH withdrawal on catalase activity}

321 Catalase activity was reduced in the brain of animals exposed to the withdrawal regime $(\mathrm{Z}=$

$3222.0885, p=0.0156$; Figure 4A), while no significant differences were found in the head kidney ( $\mathrm{Z}$ $323=1.2547, p=0.1863$; Figure 4B). Müller et al. (2017) also observed decreased catalase activity in 324 the brains of zebrafish after $24 \mathrm{~h} \mathrm{EtOH}$ withdrawal. Data and scripts for this experiment are 325 available at https://github.com/lanec-unifesspa/etoh-withdrawal/tree/master/catalase.

\section{4. Discussion}


The present work reinforced the utility of using zebrafish as a model organism in studying

329 ethanol withdrawal by searching for broader patterns in the literature, providing a conceptual

330 replication of some findings regarding anxiety-like behavior and catalase activity, as well as

331 expanding the range of behavioral domains for study. We found that the literature is inconsistent in

332 what regards the effects of ethanol withdrawal. This heterogeneity is associated with the great

333 procedure differences which are reported; the results of the metanalysis suggest that the main

334 driving factors are ethanol concentration during exposure and exposure duration, with lower

335 concentrations and longer durations more likely to induce anxiety-like behavior. Moreover, we

336 found that ethanol withdrawal (after exposing animals for 16 days to $0.5 \%$ ethanol and $1 \mathrm{~h}$

337 withdrawal) decreased scototaxis, but increased risk assessment, in the light/dark test, and

338 decreased the threshold for chemically-induced epileptic seizure-like behavior.

339 Zebrafish is increasingly being considered as a model organism in behavioral research

340 (Bonan \& Norton, 2015; Kalueff et al., 2012; Norton \& Bally-Cuif, 2010; Stewart et al., 2015), with

341 a great deal of studies on alcohol (Tran et al., 2016). Behavioral effects of drug withdrawal have

342 been demonstrated with different drugs, including cocaine (López-Patiño, Yu, Cabral, et al., 2008;

343 López-Patiño, Yu, Yamamoto, et al., 2008) and morphine (Cachat et al., 2010; Khor et al., 2011;

344 Wong et al., 2010); in all cases, anxiety-like behavior was assessed. In the same direction, ethanol

345 withdrawal has been studied mainly with models for anxiety-like behavior (Table 3). Our

346 metanalysis revealed a significant effect of $\mathrm{EtOH}$ withdrawal on anxiety-like or defensive behavior

347 in zebrafish, but a high degree of heterogeneity. Most of the heterogeneity was explained by

348 procedural aspects; significant effects of exposure duration and $\mathrm{EtOH}$ concentration were found,

349 suggesting that longer exposure and higher concentrations are critical to induce withdrawal. Other

350 factors, including statistical inference and lack of control factors (Gerlai, 2018), could influence the

351 heterogeneity as well. Most of the studies were underpowered, and there was an association

352 between observed power and effect size. Risk of bias (RoB) was very high for all studies, which 
353 usually did not report blinding and random allocation; nonetheless, study quality (i.e., RoB) did not

354 influence the results of the metanalysis, evidencing the robustness of the method. While publication

355 bias is a relevant issue in the reproducibility and replicability of zebrafish research (Gerlai, 2018),

356 no evidence for it was found in the systematic review.

357 Our conceptual replication used $60 \mathrm{~min}$ withdrawal, translationally relevant to the initial

358 symptoms of EtOH withdrawal in humans (which include anxiety symptoms and epileptic seizures;

359 Trevisan et al., 1998). Using the exposure method described in Gerlai et al. (2009), we showed that

360 withdrawal reduces scototaxis. This is in line with the "daily-moderate" condition in Holcombe et

361 al. (2013), which showed a decrease in scototaxis after using the same exposure profile we

362 presented. As can be deprehended from the meta-analysis, the effect sizes calculated for scototaxis

363 in Holcombe et al.'s (2013) experiment are similar in direction and magnitude. The Mathur and

364 Guo (2011) scototaxis study used a higher concentration of EtOH (1\%) but a shorter exposure

365 period (8 days). While Pittman and Ichikawa (2013) used a similar exposure period (14 days), the

366 concentration was much higher (3\%); in both studies, EtOH withdrawal did not affect scototaxis.

367 While following only effects on scototaxis confirms findings reported in Holcombe et al.

368 (2013), these findings contradict the overall results from the meta-analysis, which suggested that

369 EtOH withdrawal increases anxiety-like behavior in zebrafish. If other variables are considered,

370 however, the picture changes, with increases in risk assessment and number of transitions. The

371 increase in risk assessment is consistent with increased anxiety-like behavior (Maximino et al.,

372 2014), but, since scototaxis was decreased, this conclusion is only provisional. These results are

373 difficult to interpret, but could be explained by the increased risk assessment; however, an

374 exploratory analysis suggests negative correlation between risk assessment and time on white in the

375 withdrawal group ( $\mathrm{r}^{2}=-0.443$, vs. -0.122 in the control group). The increase in transitions without

376 an apparent increase in locomotion in the white compartment could be interpreted as psychomotor

377 agitation, an important symptom of EtOH withdrawal. 
A reduction in scototaxis would be expected of animals chronically exposed to EtOH, which

\section{9}

380

381

382

383

384 could decrease anxiety levels, and therefore not be a direct effect of withdrawal. While initially unplanned, we performed additional experiments to test this hypothesis by exposing a different group of animals to chronic ethanol and analyzing its behavior without withdrawal. We observed decreased scototaxis, but we also observed decreased erratic swimming and thigmotaxis. These results present preliminary evidence that withdrawal itself, and not the chronic exposure to EtOH, produced the reported behavioral effects.

In addition to this behavioral profile, $\mathrm{EtOH}$ withdrawal was also shown to decrease the threshold for chemically-induced epileptic seizure-like behavior in zebrafish. Pilocarpine is a muscarinic agonist which induces epileptic seizure-like behavior in rodents (Scorza et al., 2009), and has recently been shown to induce a similar profile in zebrafish (Pinto, 2015). The rationale of using a sub-convulsive dose is that, if $\mathrm{EtOH}$ withdrawal increases susceptibility to epileptic seizurelike behavior, zebrafish should present epileptic seizure-like behavior with a dose which does not induce this state in control animals. We observed increased probability of entering Stage 4 epileptic seizure-like behaviors in EtOH withdrawal animals, and a shorter latency to this event, suggesting that the protocol presented here is able to model susceptibility to epileptic seizure-like behavior, increasing the range of endpoints for studying $\mathrm{EtOH}$ withdrawal in zebrafish.

Finally, we also observed decreased catalase activity in the brain, but not in the head kidney of EtOH withdrawal animals. Catalase is a detoxifying enzyme that catalyzes the transformation of hydrogen peroxide, a free radical, into water and oxygen (Aebi, 1984); in the brain, catalase is poorly expressed, but usually associated with microglial activity (Dringen, 2005). The inhibition of enzymatic activity observed here replicates results by Müller et al. (2017) in the zebrafish brain; in that study, however, the exposure duration was shorter (8 days), the concentration of EtOH was higher $(1 \%)$, and the pattern of exposure was different (animals were exposed for 20 min per day, instead of continuously, to ethanol) compared to the present investigation. The lack of effect on the 
403 head kidney, where interrenal cells (the teleost functional equivalent of the mammalian adrenal

404 cortex) lie, suggesting that possible effects on cortisol (e.g., Cachat et al., 2010) are not due to

405 effects in these cells, but upstream in the hypothalamus-hypophyseal-interrenal axis.

The present work contributed to the use of zebrafish as a model in EtOH withdrawal

research by: A) identifying sources of heterogeneity in the literature on $\mathrm{EtOH}$ withdrawal and

anxiety-like behavior in the species; B) presenting a conceptual replication of withdrawal-induced

anxiogenesis in the light/dark test; C) extending the behavioral phenotypes to include epileptic

410 seizure-like behavior susceptibility; and D) replicating the effects on catalase activity, suggesting

411 that EtOH withdrawal-elicited oxidative stress could be a mechanism of anxiogenesis in the species.

412 Further work will characterize these mechanisms with care.

\section{Acknowledgements}

415 SNSC was the recipient of a CNPq/PIBIC studentship. GRF was the recipient of a CNPq/PIBITI

416 studentship. WEAO was the recipient of a CNPq/PIBIC-EM studentship to high school students.

417 This manuscript appeared as a preprint on bioRxiv (doi: 10.1101/201368).

\section{References}

Aebi, H., 1984. Catalase in vitro, in: Methods in Enzymology. pp. 121-126. doi:10.1016/S00766879(84)05016-3

Benneh, C.K., Biney, R.P., Mante, P.K., Tandoh, A., Adongo, D.W., Woode, E., 2017. Maerua angolensis stem bark extract reverses anxiety and related behaviours in zebrafishInvolvement of GABAergic and 5-HT systems. J. Ethnopharmacol. 207, 129-145. doi:10.1016/j.jep.2017.06.012

Bonan, C.D., Norton, W.H.J., 2015. The utility of zebrafish as a model for behavioural genetics. Curr. Opin. Behav. Sci. 2, 34-38. doi:10.1016/j.cobeha.2014.07.003 
Bonassoli, V.T., Milani, H., Oliveira, R.M.W. de, 2011. Ethanol withdrawal activates nitric oxideproducing neurons in anxiety-related brain areas. Alcohol 45, 641-652. doi:10.1016/j.alcohol.2010.11.007

Cachat, J., Canavello, P., Elegante, M., Bartels, B., Hart, P., Bergner, C., Kalueff, A. V, 2010. Modeling withdrawal syndrome in zebrafish. Behav. Brain Res. 208, 371-376.

Chakravarty, D.N., Faingold, C.L., 1998. Comparison of neuronal response patterns in the external and central nuclei of inferior colliculus during ethanol administration and ethanol withdrawal. Brain Res. 783, 55-57.

Conselho Nacional de Controle de Experimentação Animal - CONCEA, 2017. Diretriz brasileira para o cuidado e a utilização de animais para fins científicos e didáticos - DBCA. Anexo I. Peixes mantidos em instalações de instituições de ensino ou pesquisa científica. Brasil.

Dewari, P.S., Ajani, F., Kushawah, G., Kumar, D.S., Mishra, R.K., 2016. Reversible loss of reproductive fitness in zebrafish on chronic alcohol exposure. Alcohol 50, 83-89. doi:10.1016/j.alcohol.2015.11.006

Dringen, R., 2005. Oxidative and antioxidative potential of brain microglial cells. Antioxid. Redox Signal. 7, 1223-1233. doi: 10.1089/ars.2005.7.1223.

Egger, M., Smith, G.D., Schneider, M., Minder, C., 1997. Bias in meta-analysis detected by a simple, graphical test. Br. Med. J. 315, 629-634.

Gatch, M.B., Lal, H., 2001. Animal models of the anxiogenic effects of ethanol withdrawal. Drug Dev. Res. 54, 95-115.

Gerlai, R., 2014. Fish in behavior research: Unique tools with a great promise! J. Neurosci. Methods, 234, 54-58. doi:10.1016/j.jneumeth.2014.04.015

Gerlai, R., 2018. Reproducibility and replicability in zebrafish behavioral neuroscience research. Pharm. Biochem. Behav., In press. doi:10.1016/j.pbb.2018.02.005

Gerlai, R., 2010. Zebrafish antipredatory responses: A future for translational research? Behav. Brain Res. 207, 223-231. doi:10.1016/j.bbr.2009.10.008

Gerlai, R., Ahmad, F., Prajapati, S., Manuscript, A., Populations, A.Z., 2009. Differences in acute alcohol-induced behavioral responses among zebrafish populations. Alcohol. Clin. Exp. Res. 32, 1763-1773. doi:10.1111/j.1530-0277.2008.00761.x

Green, J., Collins, C., Kyzar, E. J., Pham, M., Roth, A., Gaikwad, S., Cachat, J., Stewart, A. M., Landsman, S., Grieco, F., Tegelenbosch, R., Noldus, L. P., Kalueff, A. V., 2012. Automated high-throughput neurophenotyping of zebrafish social behavior. J. Neurosci. Methods, 210, 266-271. doi: 10.1016/j.jneumeth.2012.07.017.

Hedges, L. V, Olkin, I., 1985. Statistical methods for meta-analysis. Academic Press, San Diego. 
Holcombe, A., Howorko, A., Powell, R.A., Schalomon, M., Hamilton, T.J., 2013. Reversed scototaxis during withdrawal after daily-moderate, but not weekly-binge, administration of ethanol in zebrafish. PLoS One 8, e63319. doi:10.1371/journal.pone.0063319

Hooijmans, C.R., Rovers, M.M., de Vries, R.B.M., Leenaars, M., Ristskes-Hoitinga, M., Langendam, M.W., 2014. SYRCLE's risk of bias tool for animal studies. BMC Med. Res. Methodol. 14, 43.

Hooijmans, C.R., Tillema, A., Leenaars, M., Ritskes-Hoitinga, M., 2010. Enhancing search efficiency by means of a search filter for finding all studies on animal experimentation in PubMed. Lab. Anim. 44, 170-175.

Hortopan, G.A., Dinday, M.T., Baraban, S.C., 2010. Zebrafish as a model for studying genetic aspects of epilepsy. Dis. Model. Mech. 3, 144-148. doi:10.1242/dmm.002139

Kalueff, A. V, Echevarria, D.J., Stewart, A.M., 2014. Gaining translational momentum: More zebrafish models for neuroscience research. Prog. Neuropsychopharmacol. Biol. Psychiatry 55, 1-6. doi:10.1016/j.pnpbp.2014.01.022

Kalueff, A. V, Gebhardt, M., Stewart, A.M., Cachat, J.M., Brimmer, M., Chawla, J.S., Craddock, C., Kyzar, E.J., Roth, A., Landsman, S., Gaikwad, S., Robinson, K., Baatrup, E., Tierney, K., Shamchuk, A., Norton, W., Miller, N., Nicolson, T., Braubach, O., Gilman, C.P., Pittman, J., Rosemberg, D.B., Gerlai, R., Echevarria, D., Lamb, E., Neuhauss, S.C.F., Weng, W., BallyCuif, L., Schneider, H., Zebrafish Neuroscience Research Consortium, 2013. Towards a comprehensive catalog of zebrafish behavior 1.0 and beyond. Zebrafish 10, 70-86. doi:10.1089/zeb.2012.0861

Kalueff, A. V, Stewart, A.M., Kyzar, E.J., Cachat, J., Gebhardt, M., Landsman, S., Robinson, K., Maximino, C., Herculano, A.M., Jesuthasan, S., Wisenden, B., Bally-Cuif, L., Lange, M., Vernier, P., Norton, W., Tierney, K., Tropepe, V., Neuhauss, S.C.F., Zebrafish Neuroscience Research Consortium, 2012. Time to recognize zebrafish "affective" behavior. Behaviour 149, 1019-1036. doi:10.1163/1568539X-00003030

Khor, B.S., Jamil, M.F., Adenan, M.I., Shu-Chien, A.C., Fadzly, M., Jamil, A., Adenan, M.I., Shu-, A.C., 2011. Mitragynine attenuates withdrawal syndrome in morphine-withdrawn zebrafish. PLoS One 6, e28340. doi:10.1371/journal.pone.0028340

Kokel, D., Peterson, R.T., 2008. Chemobehavioural phenomics and behaviour-based psychiatric drug discovery in the zebrafish. Briefings Funct. Genomics Proteomics 7, 483-490.

Koob, G.F., Le Moal, M., 2008. Neurobiological mechanisms for opponent motivational processes in addiction. Philos. Trans. R. Soc. Part B 363, 3113-3123. doi:10.1098/rstb.2008.0094

Krystal, J.H., Tabakoff, B., 2002. Ethanol abuse, dependence, and withdrawal: Neurobiology and clinical implications, in: Neuropsychopharmacology: The Fifth Generation of Progress. Lippincott, Williams \& Wilkins, Philadelphia, pp. 1425-1443. 
Kysil, E., Meshalkina, D. Frick, E. E., Echevarria, D. J., Rosemberg, D. B., Maximino, C., Lima, M. G., Abreu, M. S., Giacomini, A. C., Barcellos, L. J. G., Song, C., Kalueff, A. V., 2016. Comparative analyses of zebrafish anxiety-like behavior using conflict-based novelty tests. Zebrafish 14, 197-208. doi: 10.1089/zeb.2016.1415.

Lawrence, C., 2007. The husbandry of zebrafish (Danio rerio): A review. Aquaculture 269, 1-20. doi:10.1016/j.aquaculture.2007.04.077

Long, C., Yang, L., Faingold, C.L., Evans, M.S., 2007. Excitatory amino acid receptor-mediated responses in periaqueductal gray neurons are increased during ethanol withdrawal. Neuropharmacology 52, 802-811. doi:10.1016/j.neuropharm.2006.09.019

Longo, D.L., Schuckit, M.A., 2014. Recognition and management of withdrawal delirium (delirium tremens). N. Engl. J. Med. 371, 2109-2113. doi:10.1056/NEJMra1407298

López-Patiño, M.A., Yu, L., Cabral, H., Zhdanova, I. V, 2008a. Anxiogenic effects of cocaine withdrawal in zebrafish. Physiol. Behav. 93, 160-171. doi:10.1016/j.physbeh.2007.08.013

López-Patiño, M.A., Yu, L., Yamamoto, B.K., Zhdanova, I. V, 2008b. Gender differences in zebrafish responses to cocaine withdrawal. Physiol. Behav. 95, 36-47. doi:10.1016/j.physbeh.2008.03.021

Mathur, P., Guo, S., 2011. Differences of acute versus chronic ethanol exposure on anxiety-like behavioral responses in zebrafish. Behav. Brain Res. 219, 234-239. doi:10.1016/j.bbr.2011.01.019

Maximino, C., Brito, T.M. de, Silva, A.W.B. da, Herculano, A.M., Morato, S., Gouveia Jr, A., Marques, T., Brito, D., Waneza, A., Manoel, A., Gouveia, A., 2010. Measuring anxiety in zebrafish: A critical review. Behav. Brain Res. 214, 157-171. doi:10.1016/j.bbr.2010.05.031

Maximino, C., Benzecry, R., Matos, K. R. O., Batista, E. J. O., Herculano, A. M., Rosemberg, D. B., Oliveira, D. L., Blaser, R., 2012. A comparison of the light/dark and novel tank tests in zebrafish. Behaviour 149, 1099-1123. doi: 10.1163/1568539X-00003029

Mohammad, F., Ho, J., Lim, C.L., Woo, J.H., Poon, D.J.J., Lamba, B., Claridge-chang, A., 2016. Concordance and incongruence in preclinical anxiety models: Systematic review and metaanalyses. bioRxiv. doi:10.1101/020701

Müller, T.E., Nunes, S.Z., Silveira, A., Loro, V.L., Rosemberg, D.B., 2017. Repeated ethanol exposure alters social behavior and oxidative stress parameters of zebrafish. Prog. NeuroPsychopharmacology Biol. Psychiatry. doi:10.1016/j.pnpbp.2017.05.026

Mussulini, B.H.M., Leite, C.E., Zenki, K.C., Moro, L., Baggio, S., Rico, E.P., Rosemberg, D.B., Dias, R.D., Mello e Souza, T., Calcagnotto, M.E., Campos, M.M., Battastini, A.M.O., de Oliveira, D.L., 2013. Seizures induced by pentylenetetrazole in the adult zebrafish: A detailed behavioral characterization. PLoS One 8, e54515. doi:10.1371/journal.pone.0054515

Norton, W., Bally-Cuif, L., 2010. Adult zebrafish as a model organism for behavioural genetics. BMC Neurosci. 11, 90. doi:10.1186/1471-2202-11-90 
535 Oliveira, R.F., 2013. Mind the fish: Zebrafish as a model in cognitive social neuroscience. Front.

$536 \quad$ Neural Circuits 7, Article 131. doi:10.3389/fncir.2013.00131

Pan, Y., Chaterjee, D., Gerlai, R., 2012. Strain dependent gene expression and neurochemical levels in the brain of zebrafish transcriptome: Focus on a few alcohol related targets. Physiol. Behav. In press. doi:10.1016/j.physbeh.2012.01.017

Parra, K. V, Adrian Jr, J.C., Gerlai, R., 2009. The synthetic substance hypoxanthine 3-N-oxide elicits alarm reactions in zebrafish (Danio rerio). Behav. Brain Res. 205, 336-341. doi:10.1016/j.bbr.2009.06.037

Pinto, C.B., 2015. Caracterização do perfil de crises epilépticas e dos efeitos comportamentais induzidos por pilocarpina em peixe-zebra adulto (Doctoral thesis, Universidade Federal do Rio Grande do Sul). Retrieved from http://hdl.handle.net/10183/118000

Pittman, J., Hylton, A., 2015. Behavioral, endocrine, and neuronal alterations in zebrafish (Danio rerio) following sub-chronic coadministration of fluoxetine and ketamine. Pharmacol. Biochem. Behav. 139, 158-162. doi:10.1016/j.pbb.2015.08.014

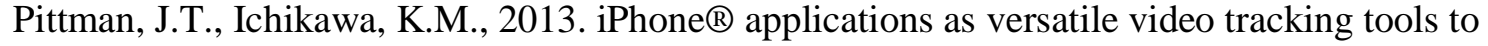
analyze behavior in zebrafish (Danio rerio). Pharmacol. Biochem. Behav. 106, 137-142. doi:10.1016/j.pbb.2013.03.013

Rasmussen, D.D., Boldt, B.M., Wilkinson, C.W., Mitton, D.R., 2002. Chronic daily ethanol and withdrawal: 3 . Forebrain pro-opiomelanocortin gene expression and implications for dependence, relapse, and deprivation effect. Alcohol. Clin. Exp. Res. 26, 535-546.

Shams, S., Rihel, J., Ortiz, J.G., Gerlai, R.T., 2018. The zebrafish as a promising tool for modeling human brain disorders: A review based upon an IBNS Symposium. Neurosci. Biobehav. Rev. $75,176-190$.

Simmons, J. P., Nelson, L. D., Simonsohn, U., 2012. A 21 Word Solution. SPSP Dialogue 26, 1-4.

Speedie, N., Gerlai, R., 2008. Alarm substance induced behavioral responses in zebrafish (Danio rerio). Behav. Brain Res. 188, 168-177. doi:10.1016/j.bbr.2007.10.031

Stewart, A.M., Ullmann, J.F.P., Norton, W.H.J., Parker, M.O., Brennan, C.H., Gerlai, R., Kalueff, A. V, 2015. Molecular psychiatry of zebrafish. Mol. Psychiatry 20, 2-17. doi:10.1038/mp.2014.128

Tran, S., Chatterjee, D., Gerlai, R., 2015a. An integrative analysis of ethanol tolerance and withdrawal in zebrafish (Danio rerio). Behav. Brain Res. 276, 161-170. doi:10.1016/j.bbr.2014.02.034

Tran, S., Facciol, A., Gerlai, R., 2016. The zebrafish, a novel model organism for screening compounds affecting acute and chronic ethanol-induced effects. Int. Rev. Neurobiol. 126, 467484. doi:10.1016/bs.irn.2016.02.016 
Tran, S., Nowicki, M., Chatterjee, D., Gerlai, R., 2015b. Acute and chronic ethanol exposure differentially alters alcohol dehydrogenase and aldehyde dehydrogenase activity in the zebrafish liver. Prog. Neuro-Psychopharmacology Biol. Psychiatry 56, 221-226. doi:10.1016/j.pnpbp.2014.09.011

Trevisan, L. A., Boutros, N., Petrakis, I. K., Krystal, J. H., 1998. Complications of alcohol withdrawal. Alcool Health Res. World 22, 61-66.

Vesterinen, H. M., Sena, E. S., Egan, K. J., Hirst, T. C., Churolov, L., Currie, G. L., Antonic, A., Howells, D. W., Macleod, M. R. Meta-analysis of data from animal studies: A practical guide. J. Neurosci. Methods 221, 92-102.

Viechtbauer, W., 2010. Conducting meta-analyses in R with the metafor package. J. Stat. Softw. 36, $1-48$.

Wong, K., Elegante, M., Bartels, B., Elkhayat, S., Tien, D., Roy, S., Goodspeed, J., Suciu, C., Tan, J., Grimes, C., Chung, A., Rosenberg, M., Gaikwad, S., Denmark, A., Jackson, A., Kadri, F., Chung, K.M., Stewart, A., Gilder, T., Beeson, E., Zapolsky, I., Wu, N., Cachat, J., Kalueff, A. V, 2010. Analyzing habituation responses to novelty in zebrafish (Danio rerio). Behav. Brain Res. 208, 450-457. doi:10.1016/j.bbr.2009.12.023

Yang, L., Long, C., Evans, M.S., Faingold, C., 2002. Ethanol withdrawal results in aberrant membrane properties and synaptic responses in periaqueductal gray neurons associated with seizure susceptibility. Brain Res. 957, 99-108.

Yang, L., Long, C., Faingold, C.L., 2001. Neurons in the deep layers of superior colliculus are a requisite component of the neuronal network for seizures during ethanol withdrawal. Brain Res. 920, 134-141.

Yang, L., Long, C., Randall, M.E., Faingold, C.L., 2003. Neurons in the periaqueductal gray are critically involved in the neuronal network for audiogenic seizures during ethanol withdrawal. Neuropharmacology 44, 275-281. doi:10.1016/S0028-3908(02)00367-2

Zenki, K.C., Mussulini, B.H.M., Rico, E.P., de Oliveira, D.L., Rosemberg, D.B., 2014. Effects of ethanol and acetaldehyde in zebrafish brain structures: An in vitro approach on glutamate uptake and on toxicity-related parameters. Toxicol. Vitr. 28, 822-828. doi:10.1016/j.tiv.2014.03.008 


\section{Tables and table legends}

600 Table 1. Operational definitions for behavioral endpoints assessed in the light/dark test. Whenever

601 available, codes used in the Zebrafish Behavioral Catalog (Kalueff et al., 2013) are provided.

\section{Behavioral endpoint Definition}

Freezing Time spent immobile, with the exception of opercular and eye movements, in seconds, observed in the white compartment (ZBC 1.68)

Erratic swimming Sharp changes in direction or velocity of swimming, associated with repeated fast acceleration bouts, observed in the white compartment (ZBC $1.51)$

Thigmotaxis Percentage of time swimming in a distance of $2 \mathrm{~cm}$ or less from the white compartment's walls (ZBC 1.173)

Risk assessment Fast ( $<1$ second) entries in the white compartment, followed by re-entry in the black compartment, or partial entries in the white compartment (i.e., the pectoral fin does not cross the midline)

603 Table 2. Behavioral phenotypes scored in the pilocarpine-induced seizure model. Scores were 604 based on Mussulini et al. (2013).

\section{Score Behavioral phenotype}

$0 \quad$ Short swim mainly in the bottom of the tank.

$1 \quad$ Increased swimming activity and high frequency of opercular movement.

2 Burst swimming, left and right movements, and erratic movements.

3 Circular movements. 
4 Clonic seizure-like behavior (abnormal whole-body rhythmic muscular contraction).

$5 \quad$ Fall to the bottom of the tank, tonic seizure-like behavior (sinking to the bottom of the tank, loss of body posture, and principally by rigid extension of the body).

$6 \quad$ Death 
606 Table 3. Studies included in the metanalysis. Studies were ordered by strain used $(B S F=$ blue

607 shortfin; WT $=$ non-specified wild-type); behavioral assay $(\mathrm{LDT}=$ light $/$ dark test; NTT $=$ novel tank

608 test); ethanol concentration during exposure ([EtOH]); exposure duration; and withdrawal duration.

609

\begin{tabular}{|c|c|c|c|c|c|}
\hline Study & Strain & Assay & [EtOH] & $\begin{array}{l}\text { Exposure } \\
\text { duration }\end{array}$ & $\begin{array}{l}\text { Withdrawal } \\
\text { duration }\end{array}$ \\
\hline Holcombe et al., 2013 & BSF & LDT & $0.2 \%$ & $21 \mathrm{~d}$ & $48 \mathrm{~h}$ \\
\hline Tran et al., 2015a & $\mathrm{AB}$ & NTT & $0.5 \%$ & $22 \mathrm{~d}$ & $1 \mathrm{~h}$ \\
\hline Mathur and Guo, 2011, study 1 & $\mathrm{AB}$ & NTT & $1 \%$ & $8 \mathrm{~d}$ & $48 \mathrm{~h}$ \\
\hline Mathur and Guo, 2011, study 2 & $\mathrm{AB}$ & LDT & $1 \%$ & $8 \mathrm{~d}$ & $24 \mathrm{~h}$ \\
\hline Mathur and Guo, 2011, study 3 & $\mathrm{AB}$ & NTT & $1 \%$ & $8 \mathrm{~d}$ & $144 \mathrm{~h}$ \\
\hline Mathur and Guo, 2011, study 4 & $\mathrm{AB}$ & LDT & $1 \%$ & $8 \mathrm{~d}$ & $168 \mathrm{~h}$ \\
\hline Pittman and Hylton, 2015 & WT & NTT & $3 \%$ & $14 \mathrm{~d}$ & $48 \mathrm{~h}$ \\
\hline Cachat et al., 2010 & BSF & NTT & $0.3 \%$ & $7 \mathrm{~d}$ & $12 \mathrm{~h}$ \\
\hline Gerlai et al., 2009, study 1 & WT & Predator avoidance & $0.25 \%$ & $22 \mathrm{~d}$ & $1 \mathrm{~h}$ \\
\hline Gerlai et al., 2009, study 2 & $\mathrm{AB}$ & Predator avoidance & $0.25 \%$ & $22 \mathrm{~d}$ & $1 \mathrm{~h}$ \\
\hline Gerlai et al., 2009, study 3 & SF & Predator avoidance & $0.25 \%$ & $22 \mathrm{~d}$ & $1 \mathrm{~h}$ \\
\hline Gerlai et al., 2009, study 4 & WT & Shoaling & $0.25 \%$ & $22 \mathrm{~d}$ & $1 \mathrm{~h}$ \\
\hline Gerlai et al., 2009, study 5 & $\mathrm{AB}$ & Shoaling & $0.25 \%$ & $22 \mathrm{~d}$ & $1 \mathrm{~h}$ \\
\hline Gerlai et al., 2009, study 6 & SF & Shoaling & $0.25 \%$ & $22 \mathrm{~d}$ & $1 \mathrm{~h}$ \\
\hline Pittman and Ichikawa, 2013, study & WT & NTT & $3 \%$ & $14 \mathrm{~d}$ & $48 \mathrm{~h}$ \\
\hline
\end{tabular}


bioRxiv preprint doi: https://doi.org/10.1101/201368; this version posted March 19, 2018. The copyright holder for this preprint (which was not certified by peer review) is the author/funder, who has granted bioRxiv a license to display the preprint in perpetuity. It is made available under aCC-BY-ND 4.0 International license.

\begin{tabular}{lllll}
\hline Pittman and Ichikawa, 2013, study WT & LDT & $3 \%$ & $14 \mathrm{~d}$ & $48 \mathrm{~h}$
\end{tabular}

2

\begin{tabular}{|c|c|c|c|c|c|}
\hline Müller et al., 2017 & BSF & Shoaling & $1 \%$ & $8 \mathrm{~d}$ & $24 \mathrm{~h}$ \\
\hline Benneh et al., 2017, study 1 & WT & NTT & $0.5 \%$ & $8 \mathrm{~d}$ & $96 \mathrm{~h}$ \\
\hline Benneh et al., 2017, study 2 & WT & NTT & $0.5 \%$ & $8 \mathrm{~d}$ & $192 \mathrm{~h}$ \\
\hline Benneh et al., 2017, study 3 & WT & LDT & $0.5 \%$ & $8 \mathrm{~d}$ & $96 \mathrm{~h}$ \\
\hline Benneh et al., 2017, study 4 & WT & LDT & $0.5 \%$ & $8 \mathrm{~d}$ & $192 \mathrm{~h}$ \\
\hline Dewari et al., 2016 & WT & NTT & $0.5 \%$ & $63 \mathrm{~d}$ & $1512 \mathrm{~h}$ \\
\hline
\end{tabular}

610 


\section{Figure legends}

\section{Figure 1 - Metanalysis of EtOH withdrawal experiments in zebrafish reveal a significant}

613 increase in anxiety-like behavior, but high heterogeneity driven by methodological

614 differences. (A) Forest plot showing the results of 16 studies examining the effect of ethanol

615 withdrawal on zebrafish anxiety-like behavior. The figure shows the standardized mean difference

616 (SMD) between control and withdrawal-exposed groups with corresponding 95\% confidence

617 intervals in the individual studies, based on a mixed-effects model. A negative standardized mean

618 difference (SMD) corresponds to decreased anxiety-like behavior, while a positive SMD

619 corresponds to increased anxiety-like behavior after ethanol withdrawal. Studies are ordered by

620 total degrees of freedom. (B-D) Permutation distribution of the test statistic for the mediators:

621 behavioral test (B), ethanol concentration during exposure (C), exposure duration, in days (D),

622 withdrawal duration, in hours $(\mathrm{E})$, and strain/phenotype (F). Distributions were based on a

623 permutation test with 1,000 replications. The blue contour represents kernel density estimates of the

624 permutation distributions; the red curve represents the standard normal density; the full line

625 represents the null hypothesis of no difference; and the dashed line represents the observed values

626 of test statistics. (G) Contour-enhanced funnel plot of meta-analysis. Estimated standardized mean

627 differences were plotted against precision (1/standard error) were A negative estimate corresponds

628 to decreased anxiety-like behavior, while a positive estimate corresponds to increased anxiety-like

629 behavior after ethanol withdrawal. The unshaded region corresponds to p-values greater than 0.1 ,

630 the gray-shaded region to p-values between 0.1 and 0.05 , the dark gray-shaded region corresponds

631 to p-values between 0.05 and 0.01 , and the region outside of the funnel corresponds to p-values

632 below 0.01 .

633

634 Figure 2 - Effects of EtOH withdrawal (increasing concentration of up to $0.5 \%$ for 16 days,

635 followed by $1 \mathrm{~h}$ withdrawal) on behavior in the light/dark test. (A) Scototaxis; (B) Erratic 
636 swimming; (C) Risk assessment; (D) Freezing; (E) Thigmotaxis; (F) Transitions to white; (G)

637 Locomotion on white. Red dots represent mean, and red error bars represent nonparametric

638 bootstrapped confidence intervals for the mean at the $95 \%$ level. To facilitate visualization, data

639 points were jittered; therefore, their absolute position does not reflect the actual value, and values

640 can appear to be below 0.

642 Figure 3 - Effects of EtOH withdrawal (increasing concentration of up to $0.5 \%$ for 16 days, 643 followed by 1 h withdrawal) on Score IV seizure latencies after sub-convulsive pilocarpine

644 injection. Animals were observed after injection of $150 \mathrm{mg} / \mathrm{kg}$ pilocarpine in controls (green lines)

645 and withdrawal (red lines). The dashed lines represent 95\% confidence intervals around the Kaplan-

646 Meier estimates of seizure probability at each time interval.

648 Figure 4 - Effects of EtOH withdrawal (increasing concentration of up to $0.5 \%$ for 16 days,

649 followed by 1 h withdrawal) on catalase activity. Enzyme activity was assessed in (A) brain and

650 (B) head kidney of zebrafish from control (CTRL) and withdrawal (WD) groups. Red dots represent

651 mean, and red error bars represent nonparametric bootstrapped confidence intervals for the mean at

652 the $95 \%$ level. To facilitate visualization, data points were jittered; therefore, their absolute position

653 does not reflect the actual value, and values can appear to be below 0 .

655 Figure S1 - Influential study analysis for the metanalysis. Statistics represent standardized 656 residuals (rstudent), DFFITS (dffits), Cook's distances (cook.d), covariance ratios (cov.r), estimates 657 of $\tau^{2}$ (tau2.del) and test statistics (QE.del) for (residual) heterogeneity when each study is removed 658 in turn, hat values, and weights for each of the 16 studies examining the effects of EtOH withdrawal 659 on zebrafish defensive behavior. Red filling indicates an influential study. 
661 Figure S2 - Relationship between observed power and modulus SMD values in the metanalysis.

662 Curve fitting was made using a linear model.

663 Figure S3 - Effects of chronic ethanol exposure on behavior in the light/dark test. (A)

664 Scototaxis; (B) Erratic swimming; (C) Risk assessment; (D) Freezing; (E) Thigmotaxis; (F)

665 Transitions to white; (G) Locomotion on white. Red dots represent mean, and red error bars

666 represent nonparametric bootstrapped confidence intervals for the mean at the 95\% level. To

667 facilitate visualization, data points were jittered; therefore, their absolute position does not reflect

668 the actual value, and values can appear to be below 0 .

669

670

671 


Holcombe et al., 2013
Tran et al., 2015
Mathur et al., 2011.1
Mathur et al., 2011.2
Pittman et al., 2015
Cachat et al., 2010
Gerlai et al., 2009.1
Gerlai et al., 2009.2
Gerlai et al., 2009.3
Gerlai et al., 2009.4
Gerlai et al., 2009.5
Pittman \& Ichikawa, 2013.1
Pittman \& Ichikawa, 2013.2
Müller et al., 2017
Mathur et al., 2011.3
Mathur et al., 2011.4
Benneh et al. 2017.1
Benneh et al. 2017.2
Benneh et al. 2017.3
Benneh et al. 2017.4
Dewari et al, 2016

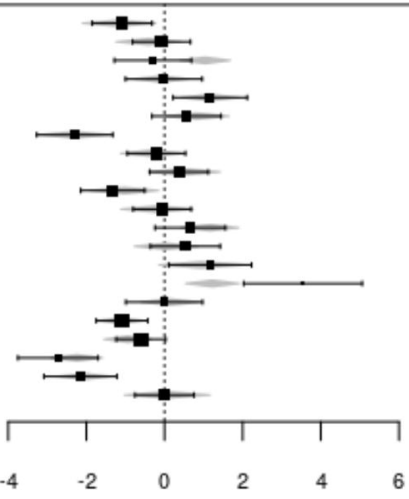

$-1.08[-1.85,-0.32]$

$-0.08\}-0.82,0.66$

$02-1.00,0.69$

$1.17\} 0.22,2.11$

$-2.30-3.27,-1.33$

$-0.21-0.95,0.53$

$0.37-0.38,1.12$

$-0.06[-0.80,0.68$

$0.66-0.24,1.5$

1.17
3.54

0.01 - $0.99 ; 0.97$

$-0.60-1.23,0.03$

$-2.72[-3.75,-1.70$

$0.00[-0.75,0.75]$

B

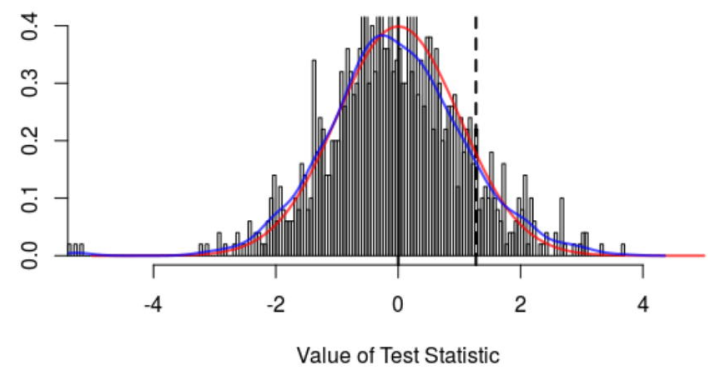

D

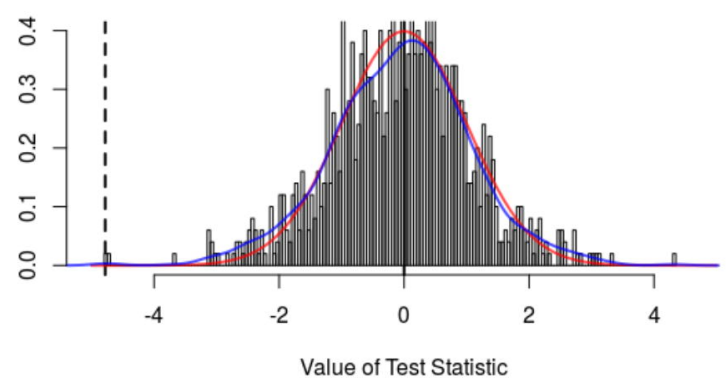

$\mathrm{F}$

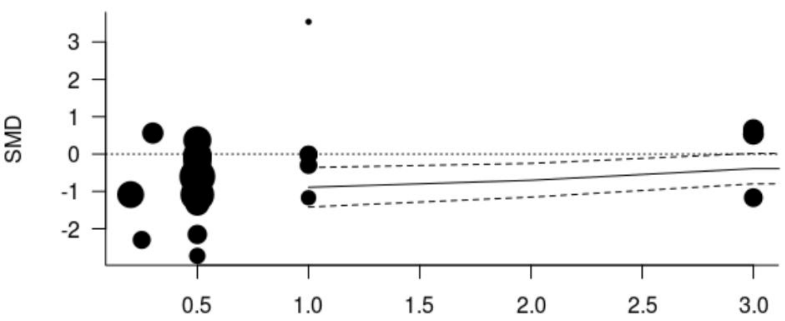

C

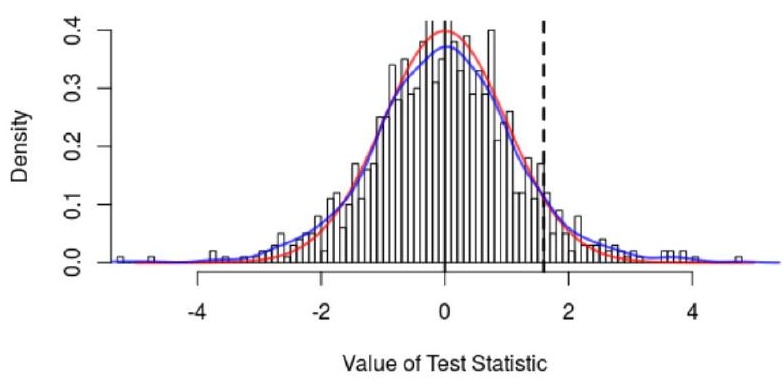

E

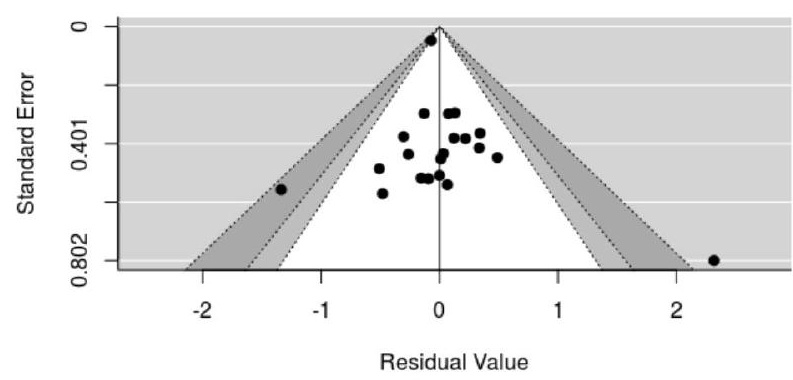

G

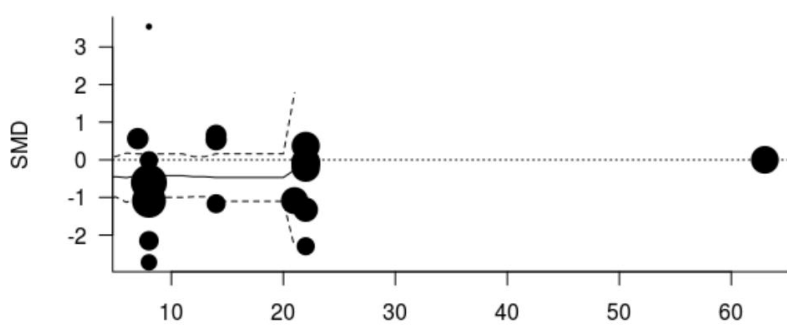



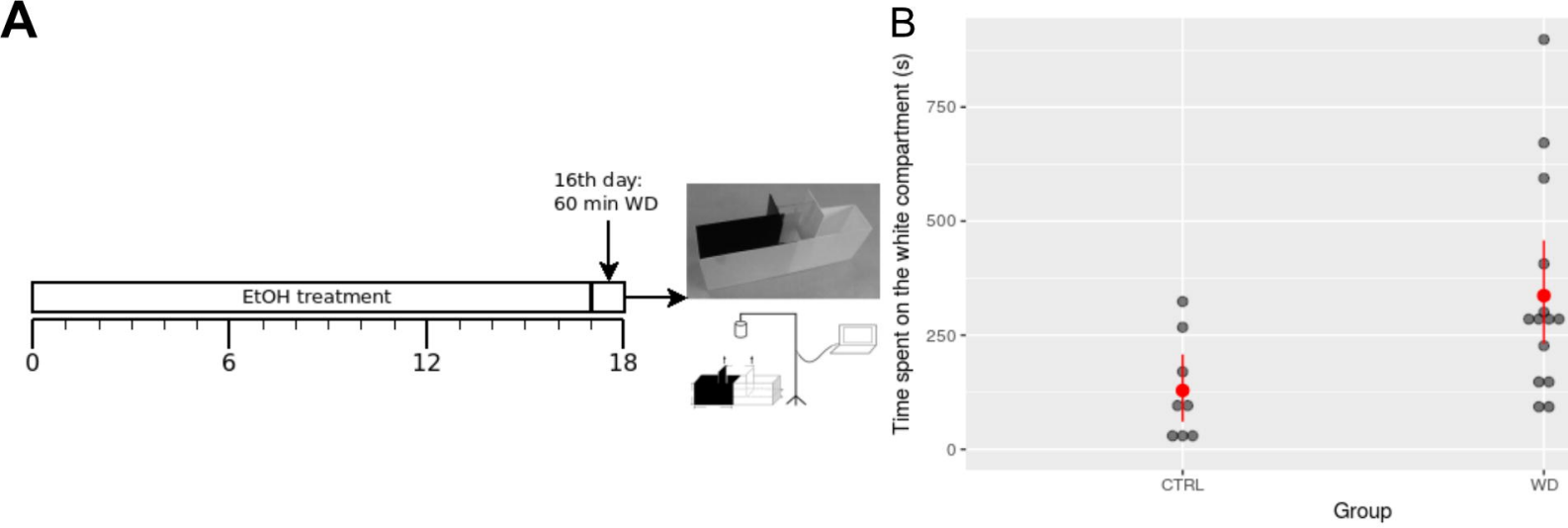

C

D

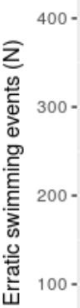

CTRL

พ่อ

$\therefore$

CT'RL

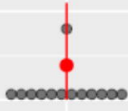

W'D

Group

F
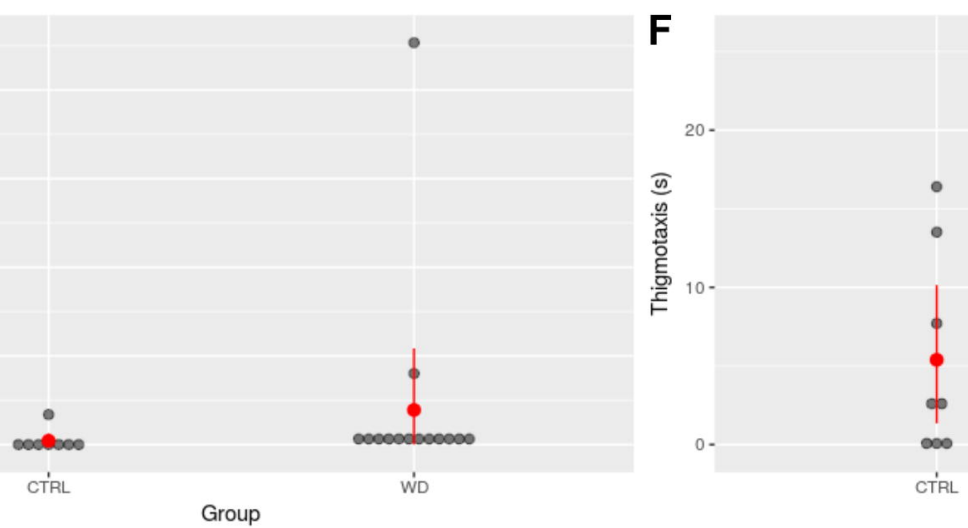

。
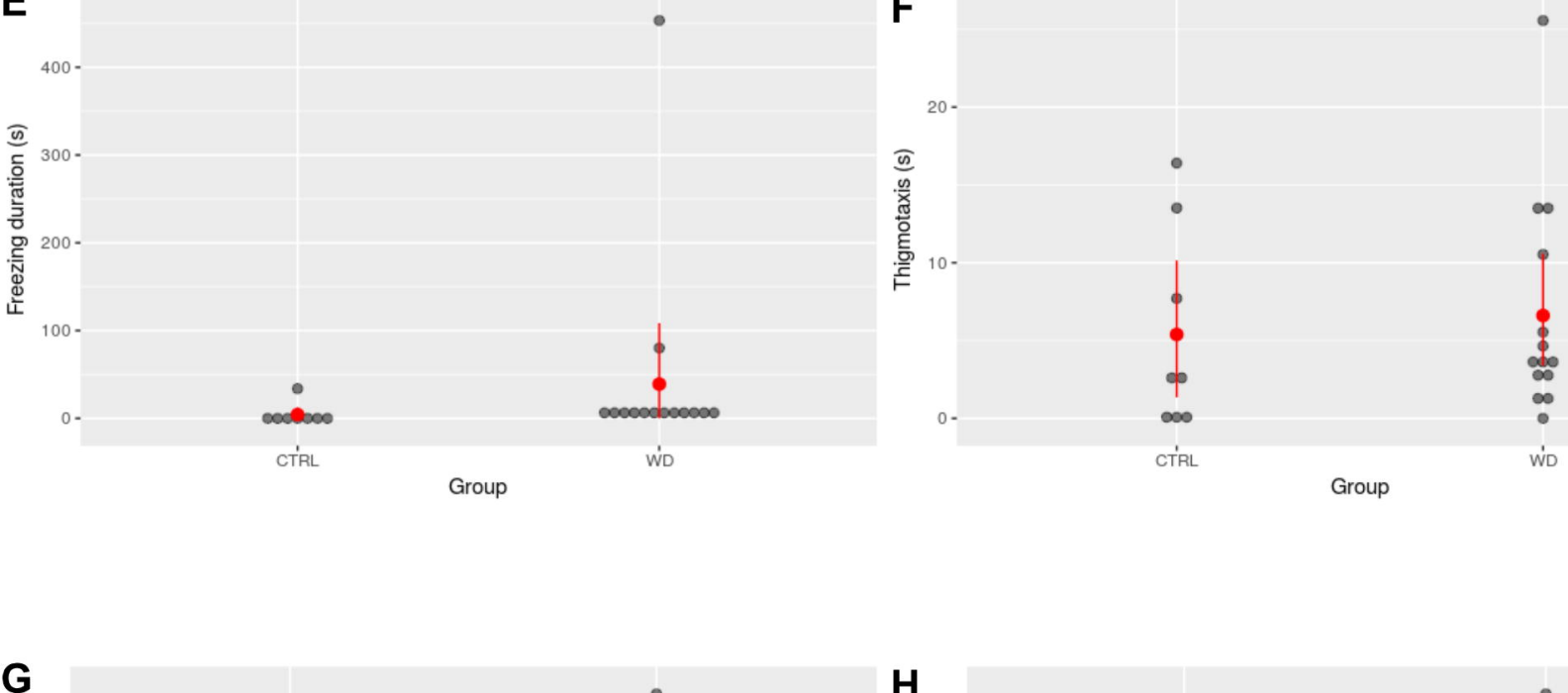

G
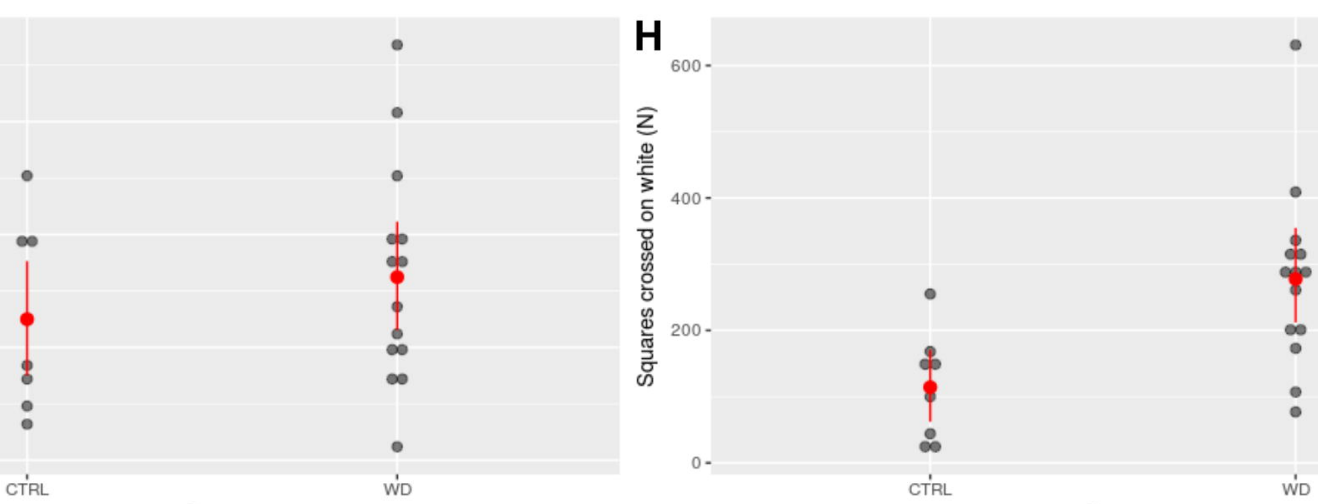


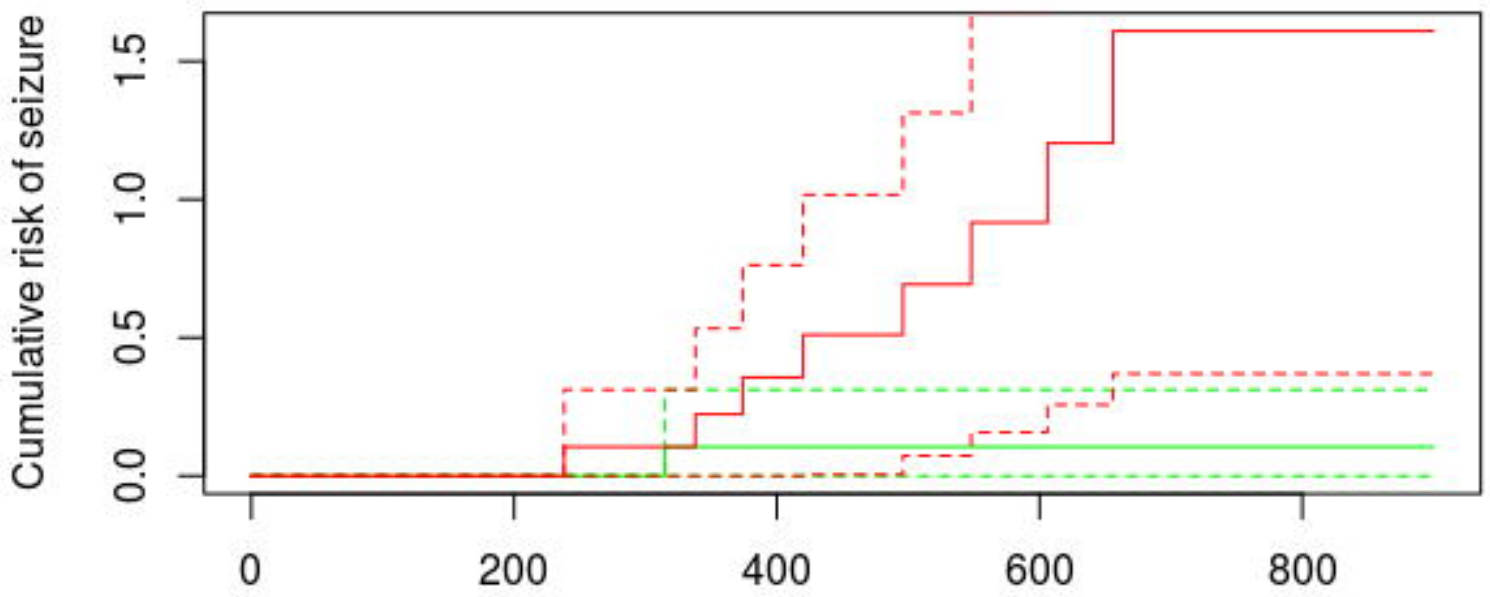

Time (s) 
